\title{
La herencia científica del Instituto Eduardo Torroja en Bilbao (1960-1980)
}

\author{
The scientific legacy of the "Instituto Eduardo Torroja" in Bilbao \\ (1960-1980)
}

L. Bilbao $^{(*)}$

\section{RESUMEN}

A través de este análisis, hemos tratado de valorar y comprender tanto la influencia que suscitó el IETcc en el progreso científico bilbaíno fundamentalmente durante los años 60 y los años 70, así como la decidida pretensión de la élite financiera e industrial local de hacer entonces de Bilbao la gran metrópoli del norte, es decir, la capital económica de aquella España, tomando para ello como modelo, entre otras metrópolis, a la ciudad americana de las finanzas, Nueva York, y a la de la industria, Pittsburgh. Se han estudiado los instrumentos de los que se valieron para que así fuera: desde organismos de investigación, exposición e información que originarían un anhelado progreso científico, así como las líneas para reproducir la fascinante vía americana.

Palabras clave: IETcc; EE.UU.; progreso; ciencia; modernidad.

\section{SUMMARY}

By means of this analysis, we have endeavored to evaluate and understand both the influence that the IETcc started to bear on scientific progress in Bilbao, mainly during the 1960s and the 1970s, as well as the determined efforts of the local financial and industrial elite of the time to transform Bilbao into the great metropolis of the north, i.e. the economic capital of that part of Spain, using as a model, among other metropolises, New York, the financial capital of America, and Pittsburg, the industrial capital. The various means to accomplish this have been studied: from the institutions of investigation, exposition and information which would initiate the longed for scientific progress, to the pathways for reproducing the fascinating American way.

Keywords: IETcc; USA; progress; science; modernity.

(*) UPV-EHU, Leioa (España).

Persona de contacto/Corresponding author: luis.bilbao.larrondo@gmail.com (L.Bilbao)

Cómo citar este artículo/Citation: Bilbao, L. (2014). La herencia científica del Instituto Eduardo Torroja en Bilbao (1960-1980). Informes de la Construcción, 66(533): e011, doi: http://dx.doi.org/10.3989/ic.12.097.

Licencia/License: Salvo indicación contraria, todos los contenidos de la edición electrónica de Informes de la Construcción se distribuyen bajo una licencia de uso y distribución Creative Commons Reconocimiento no Comercial 3.o. España (cc-by-nc). 


\section{INTRODUCCIÓN}

Tratar de valorar y comprender la influencia que suscitó el ingeniero D. Eduardo Torroja y el Instituto Técnico de la Construcción, en el conocimiento científico español, se torna no solo en un acto de obligado cumplimiento sino que también en uno de auténtico compromiso intelectual para cualquier estudioso de la historia de la arquitectura y de la construcción contemporánea. No obstante, puede resultar dicha pretensión una tarea a la vez que extremadamente complicada difícilmente viable cuando constatas su enorme transcendencia puesto que supera cualquier idea preconcebida.

Sostener algún calificativo sobre la trayectoria científica e intelectual de D. Eduardo Torroja se antoja una pretensión sumamente compleja dada su extraordinaria categoría, a su conocida faceta de ingeniero de caminos, canales y puertos de formación, hemos de unir que fue profesor de la Escuela Superior de Especialidad y Director del Laboratorio Central de Ensayos de Materiales. Detentó entre otros importantes cargos el de consejero de Obras Públicas, Director del ITcc, miembro del CSIC, Vocal del Consejo del Instituto de Racionalización, Presidente de las Comisiones de Normalización de Ensayos de Materiales de la Ingeniería Civil y de las Industrias de la Construcción, Presidente de la Federación Internacional del Hormigón Pretensado, y fundador-presidente de la Federación Internacional de Estructuras Laminares. Tal y como sostuvo la profesora Pepa Cassinello el ingenio de su modelo de pensamiento convirtió no solo su obra sino su anhelo ideológico en el camino del progreso protagonizando la revolución científica y técnica, que contribuyó más que a evolucionar, a revolucionar, sobre todo, la industria de la construcción. Si el brillante arquitecto Richard Neutra lo llegó a considerar como una personalidad excepcional en el campo de la ingeniería, o el propio arquitecto, Fernando Cassinello, lo tachó de guía del estructuralismo constructivo, provisto de una prodigiosa intuición e improvisación, pero sería la profesora Pepa Cassinello la que lo describió con una visión más completa, como un personaje reconocido internacionalmente como proyectista, científico, investigador, gestor, docente, pero siendo su escuela y la revista Informes de la Construcción, su legado más importante (1) (2) (3).

La escuela, es decir, su herencia o transmisión más importante, sirvió de modelo exportable a otras ciudades -tal y como lo fue para Bilbao-, tanto material como intelectualmente, empezando por sostener la mimetización de la estructura de su escuela, que se sustentaba en varios departamentos: Química, Materiales, Metrología, Construcción, Normativa e Información, Publicaciones y Estudios. Su finalidad no fue otra que buscar, encontrar y transmitir los conocimientos a través del estudio y la investigación, sobre los materiales de la construcción, de las técnicas de aplicación y empleo, siempre mejorando, desarrollando e innovando. También trataba de optimizar al máximo el nivel de formación y especialización de los técnicos, representando a España en foros internacionales e informaba de los hallazgos en la investigación científica y técnica, sobre todo, de la construcción y de sus materiales. Entre sus cometidos, estaba el de sustentar la investigación científico técnica, la asistencia técnica, la normalización, el control de calidad e idoneidad técnica además de la docencia e información.

En cuanto a su faceta de asistencia técnica a la iniciativa, tanto pública como privada, lo hacía a través de expedientes, informes, asesoramiento, análisis y ensayos de materiales, elementos y estructuras, de manera especial el estudio, redacción y actualización de instrumentos, normas y pliegos de condiciones así como en el control de la calidad, en la expedición de documentos de idoneidad técnica y en la homologación de materiales.

Entre las importantes herramientas de información y divulgación con las que se dotó, estaban aquellas publicaciones científicas con una singular trascendencia, como fueron además de la destacada Informes de la Construcción, Cuadernos de Investigación, Cartillas de Hormigón, Materiales de la Construcción, monografías del IETcc, manuales, folletos, libros de texto para los alumnos y el Boletín de Información Trimestral (4).

Aunadas a dichas herramientas materiales se expresaban toda una serie de, más que características premisas científicas e intelectuales, que se repitieron, una y otra vez, desde el instituto, a lo largo de los años 50 y 60: la necesidad de suscitar una innovadora línea de estrategia científica con una mayor intensificación investigadora, para lo cual dicha pretensión se debía de sustentar en la divulgación pública de las nuevas técnicas, sistemas y materiales que fueran surgiendo. Pero para que dicho progreso tuviera éxito, sostuvieron desde la escuela que, además de emular estos instrumentos materiales, se hacía preciso sostener una postura de vanguardia en el conocimiento, además de difusión e inquietud por la ciencia, de fomentar en la propia sociedad, la curiosidad por los avances científicos, el orgullo por los éxitos logrados, por medio de la investigación, creando un interés permanente, una lucha contra el analfabetismo existente (5) (6).

Los tres pilares sobre los que se sustentaría dicha innovadora política científica, aplicable a cualquier otra ciudad española, -como fue el caso de Bilbao-, pasaban por la necesidad de establecer además de un organismo de ensayos e investigación, otro sería de información-divulgación, y un tercero de exposición. Junto a estos anhelos llegaron aquellos innovadores ideales científicos, propuestas tecnológicas, políticas constructivas y toda una simbología de la modernidad, desde los Estados Unidos, que influirían, tanto en el propio IETcc (que hizo de nexo de unión entre ambos ámbitos) como en la ciudad de Bilbao.

El entonces Instituto Técnico de la construcción y el cemento, le dotaría a la ciudad de Bilbao no solamente de una mayor conciencia científica como instrumento de progreso en el ámbito industrial sino que también de los mecanismos necesarios para desarrollar una innovadora política tecnológica.

\section{LOS TRES FUNDAMENTOS DEL PROGRESO CIENTÍFICO EN BILBAO}

El primero de los fundamentos a aplicar, dentro del programa que sustentaba el ITcc como necesario para suscitar un progreso científico, pasaba por crear unos laboratorios como organismo de ensayos e investigación- y dicha premisa científica tuvo una respuesta desde la Escuela de Ingenieros de Bilbao en los años 50, cuyos laboratorios se ubicarían en la zona de Olaveaga y tomaría el nombre del ingeniero Leandro José Torrontegui, director de la escuela y artífice de su modernización. Otra de las premisas del ITcc, que cumplieron desde estos laboratorios bilbaínos, fue la de visitar aquellas instituciones y laboratorios de mayor prestigio europeo con 
el fin de analizar sus experiencias y estudiar sus progresos científicos técnicos y constatar si sus estructuras y políticas eran adaptables a Bilbao. Entre otros destacados centros se encontraban el IRSID de París, la Escuela Técnica Superior de Delft en Holanda, el Instituto Max Planck de Göttingen, el politécnico de Zurich o el laboratorio Federal de Ensayo de Materiales suizo (Figura 1).

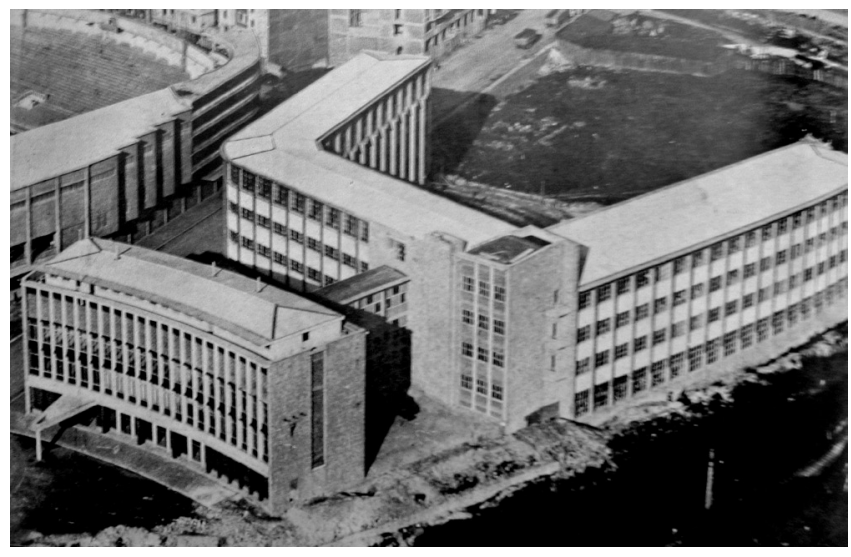

Figura 1. Escuela de ingenieros. (Archivo Municipal de Bilbao).

Las relaciones que se suscitaron en los años del Desarrollismo entre el ya Instituto Eduardo Torroja de la construcción y el cemento y los Laboratorios Torrontegui, llegaron de la investigación compartida sobre distintos sistemas y materiales, desde muros de ladrillo, normas de acciones de edificación, acero laminado, fontanería, acústica, prefabricación o de la participación en diferentes foros, cursos, ciclos de conferencias, coloquios sobre innovadores materiales o sistemas tecnológicos, en los que tomaron parte técnicos de ambas instituciones, desde el IETcc, serían los arquitectos Fernando Cassinello y Fernando Aguirre de Yraola.

Los laboratorios Torrontegui trataron de mantenerse en la vanguardia de la investigación no solo en el departamento de la construcción -control de obras, mecánica de suelos, aglomerantes, materiales metálicos y ensayos de estructurassino en el de química con sus actividades de análisis de los materiales de la construcción, o en el de electricidad y electrónica, con ensayos sobre todo tipo de equipos y aparatos, entre otros apartados.

Otra de las facetas sostenidas por el IETcc que cumplirían desde los laboratorios Torrontegui fue que frente a la necesidad de sustentar los suficientes instrumentos divulgativos con los que cumplir la premisa que resultaba vital para un organismo investigador, dado que lo más importante era asegurar la máxima difusión y aplicación posible a los resultados de sus trabajos. Después de 10 años de existencia de los laboratorios además de sus memorias empezó a editar la revista Ensayos e Investigación, que tuvo como objetivos no solo la difusión científica sino mantener un mayor contacto entre los laboratorios y la industria vizcaína con el fin de viabilizar un proyecto de futuro en avances tecnológicos (7) (8) (9).

El siguiente instrumento que proponía el IETcc sería el de divulgación (más allá de las revistas, memorias, editoriales que traducían al castellano obras emblemáticas de la ciencia constructiva) entre los que sobresaldría el diario bilbaíno
Hierro por su aportación científica, publicando a través de unas series, todos los avances conocidos en el mundo de la investigación constructiva, arquitectónica y urbanística, con sus correspondientes teorías, reflexiones, debates y controversias tecnológicas. Todo ello a través no solo de artículos de opinión sino que también de columnas críticas, monografías, fotos, representaciones gráficas... (Figura 2).

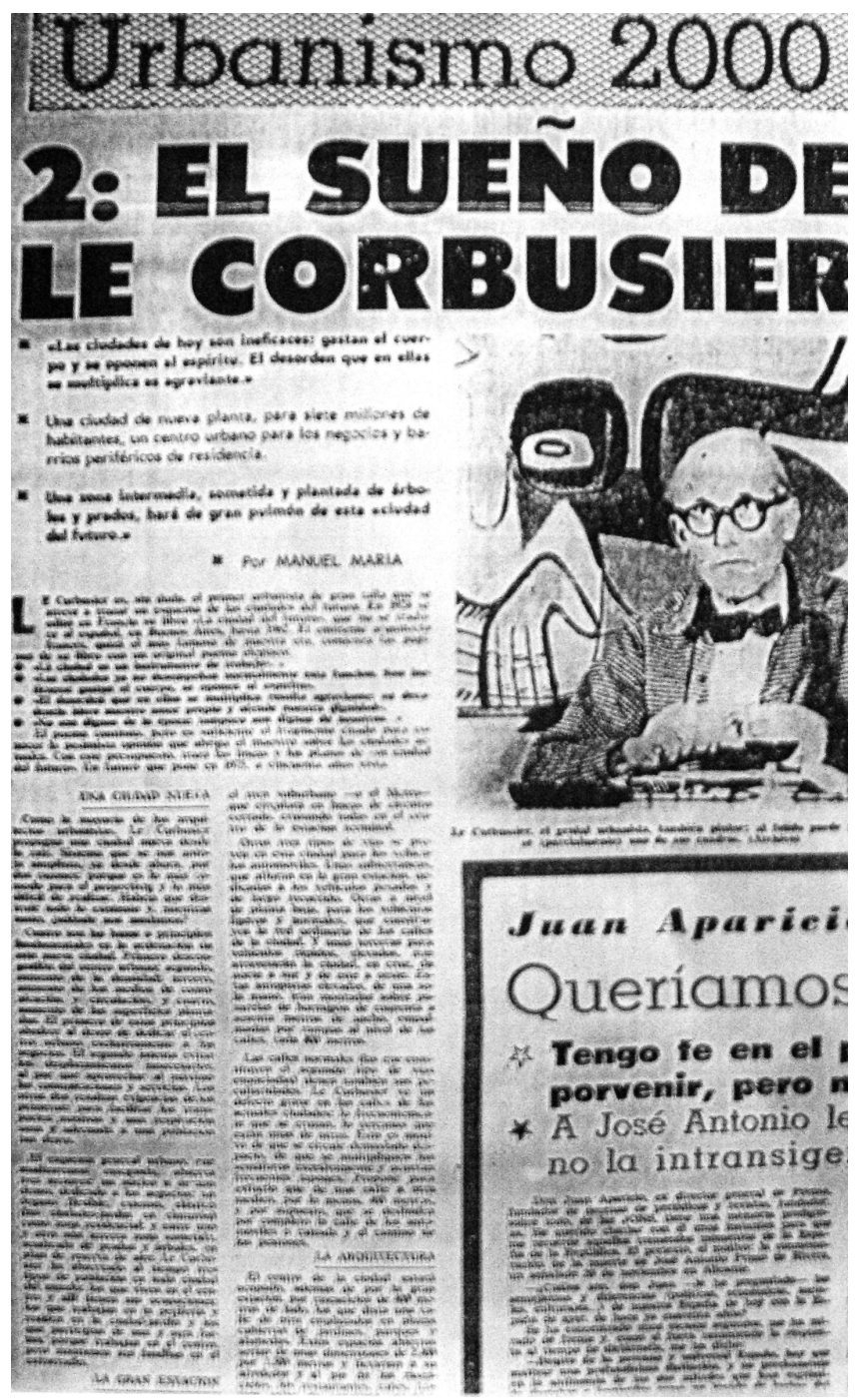

Figura 2. Diario Hierro, Serie Urbanismo 2000

Una vez analizados los numerosos artículos publicados durante estos 20 años por el diario Hierro, es cuando adviertes varias cuestiones importantes a tener en consideración, entre otras, que las fuentes a las que recurrieron eran de enorme prestigio en el mundo científico, a las provenientes del Ministerio de Industria o de la Vivienda, se aunaban instituciones como el Patronato Juan de la Cierva, el CSIC, el IETcc, publicaciones como la Gaceta de la Construcción, 3E, Focitec o el Monitor de Obras Públicas. Unidos a los prestigiosos nombres de los autores que firmaban los artículos publicados o las ideas sustentadas en dichos escritos, de ingenieros, arquitectos e investigadores de la talla de Marcel Lods, Max Abramovitz, Mathias Goeritz, Frederic Kiesler, Rene Sarger, Robert Lourdin, Victor Lundy, Yves Magnnant, Jonel Schein, Atestan Spilhaus, Jean Louis Chaneac, Paul Mosmont, Yona Friedman, Konstantin Doxiadis, Roger Coulon, Buckmeinster Fuller, Louis Armand o Jacques Riboud, entre otros. Sus 
investigaciones y publicaciones se sustentaban sobre innovadores materiales, estructuras prefabricadas, sistemas constructivos, vivienda, modelos, la ciudad y sus problemas o la industrialización de la construcción... (Figura 3).

Todo ello suscita cuando menos una enorme incertidumbre ante unos artículos cuyos temas de contenido y la calidad de los mismos estaban más cercanos a una revista profesional que a un diario de tirada local, teniendo en cuenta que esta información llegaba a manos del usuario de la calle deudor de un desconocimiento absoluto en estas cuestiones, -teorías, propuestas, reflexiones, debates, controversias y actuaciones- que se estaban produciendo en el resto del mundo en ámbitos de la ciencia y de la investigación en torno a la arquitectura, urbanismo y la construcción fundamentalmente. Resulta tanto más extraño aún si tenemos en cuenta que los años 60 a pesar de ser los años del Desarrollismo, los índices de pobreza cultural en los que estaba sumido el País Vasco, seguían siendo extraordinarios (10) (11) (12).

Además de los instrumentos u organismos de ensayos e investigación y divulgación, faltaba el de exposición, para cumplir con los axiomas sostenidos por el IETcc en el pretendido progreso tecnológico, pero no solo de los materiales, sistemas, tecnologías y maquinarias innovadoras sino que también el de las ideas, los debates y las controversias científicas. En Bilbao dicha pretensión la asumió la Feria de Muestras (Figura 4).

Con el paso de los años se consolidó como lugar de encuentro -entre especialistas, industriales y técnicos- exponiendo sus

\section{I ODI I Las ciudades crecen a un ritmo espectacular}

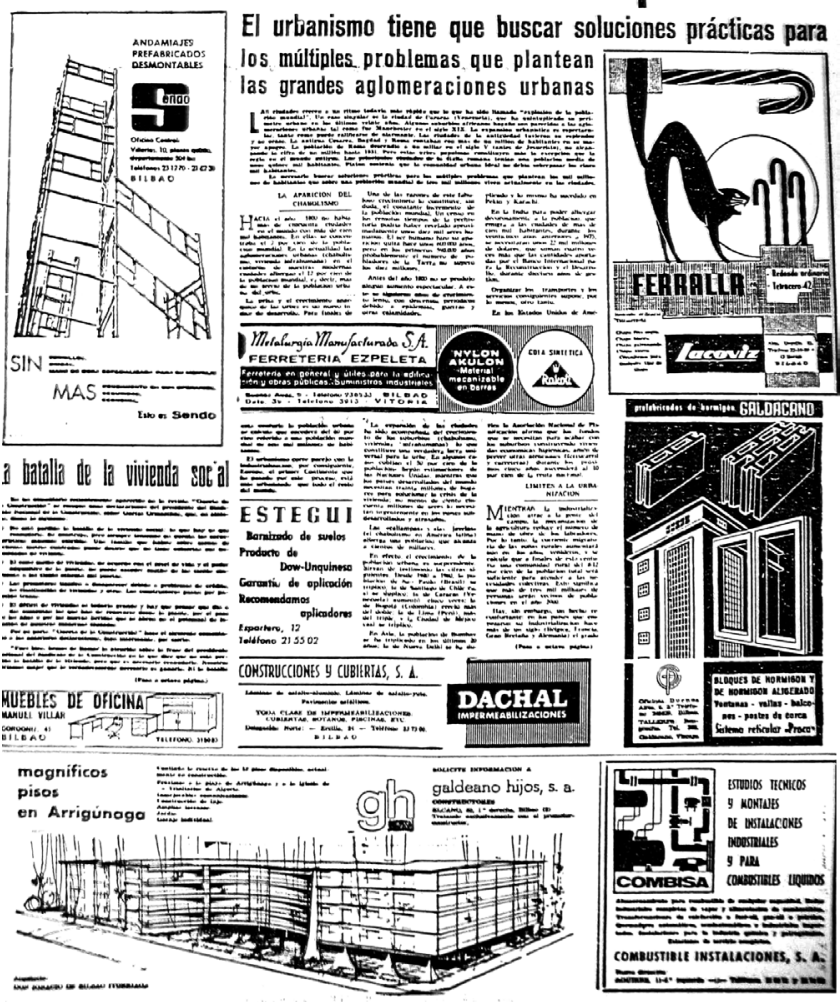

Figura 3. Diario Hierro, Serie La Obra.

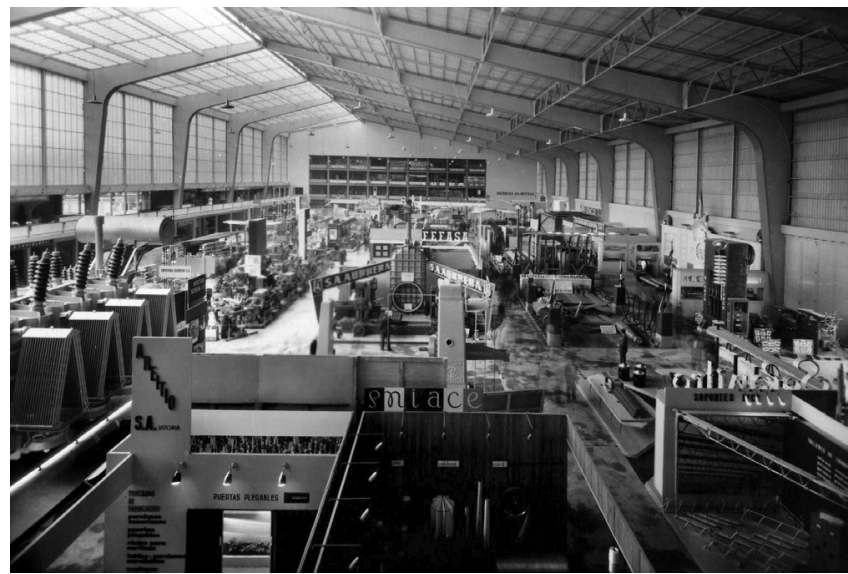

Figura 4. Feria de muestras de Bilbao, interior. (Archivo Municipal de Bilbao).

anhelos e ideas a través de films, sustentando una propaganda de las innovaciones que se suscitaban desde sus stands, catálogos de productos estandarizados, incluso como centro de experiencias. Ejemplos de las tecnologías que se exponían llegadas de Italia o Suecia, se unían los prototipos de viviendas de Francia o cocinas totalmente automatizadas que eran sustentadas por semanarios como Elle y provenientes de la Feria de Arte Domestico de Paris. En los stands aparecían los avances significativos en el interior de las viviendas a través de los cambios suscitados por la resistencia eléctrica, o los stands de Ade o Sinco, verdaderos centros informativos de la construcción. También debemos destacar su faceta como vehículo difusor de ideas, debates en torno a la arquitectura, que acogió las conferencias de arquitectos tan reconocidos como Pedro Bidagor, Javier Carvajal, Rafael Leoz o Ricardo Olaran -en torno a la Ekistica, el pabellón español de la feria de Nueva York, el sistema HELE o los sistemas de la prefabricación en la construcción-. Sin obviar, su otra faceta, la de expositor de maquetas de proyectos, viviendas, infraestructuras o de trascendentales exposiciones, como la de Roma Interrotta, que trataba de generar un debate entre la sociedad bilbaína sobre la necesidad de romper con el pasado y rehabilitar la ciudad histórica frente al desagrado generalizado ante la ciudad contemporánea (Figura 5) (13) (14) (15).

Tras analizar y valorar las importantes premisas sostenidas por el IETcc, es cuando adviertes que se viabilizó de mane-

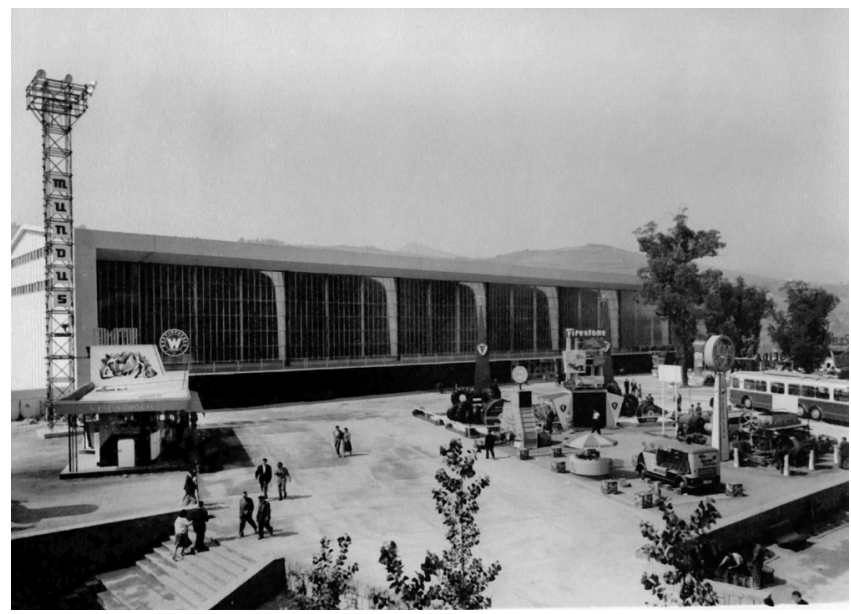

Figura 5. Feria de muestras, exterior. (Archivo Municipal de Bilbao). 
ra categórica aquella pretendida vía científica que debían de emprender y desarrollarse durante el Desarrollismo para suscitar un mayor progreso tecnológico en Bilbao. Faltaba por aunar otra vía determinante en el camino emprendido: la experiencia americana.

\section{EL IETCC Y LOS ESTADOS UNIDOS: MÍMESIS EN BILBAO ¿ÉXITO O FRACASO?}

La siguiente de las vías a analizar era la influencia que tuvo los Estados Unidos. El profesor Eduardo Torroja, gran defensor de aquellos preceptos, tuvo un singular nexo de unión con los ideales científicos que llegaban e intercambiaban con los Estados Unidos, a su labor docente en universidades como la de Raleigh, Harvard, Princeton o el MIT -con cursos de especialización sobre la investigación técnica de la construcción así como numerosas conferencias- hemos de aunar las relaciones que mantuvo con instituciones de investigación como la American Concrete Institute, American Society for Testing Materials, American Society for Heating and Ventiangting Engineers o la American Welding Society (16).

Incluso la abundante bibliografía que su escuela tiene actualmente en sus archivos de revistas, libros y otro tipo de publicaciones, sobre la construcción, ingeniería y arquitectura americana de aquellos años, fundamentalmente, es evidencia más que suficiente de aquella no solo fascinación que produjeron los avances tecnológicos suscitados en aquel país sino que también la línea científica pretendida por la escuela ( $A r-$ chitectural Forum, Architectural Records, House and Home, Progressive Architecture, International Science and Technology, Iron and Steel Enginners, Western Construction o Public Works entre otras muchas interesantes publicaciones) ${ }^{1}$.

No podemos olvidar el impulso dado a aquellas relaciones a través del Programa de Intercambio Técnico firmado en 1953 entre España y Estados Unidos (ICA) -International Corporation Administration-, siendo a partir de 1956 cuando la Comisión Nacional de Productividad Industrial organizó los viajes de información industrial dentro del programa de ayuda técnica a los Estados Unidos. A su vuelta, se editaron los estudios realizados, en unas memorias sustentadas por el Ministerio de Industria, con temas tan heterogéneos como los "Proyectos de viviendas y urbanismo de los Estados Unidos", "Materiales y métodos de construcción", "Construcción de obras", "El ladrillo cerámico", "La industria del cemento", "Memoria de estudios de tiempos y movimientos en los Estados Unidos", "Memoria de la organización y mecanización de las oficinas de los Estados Unidos", "Memoria de las Relaciones Humanas", "Memoria de la Dirección de Personal", "Memoria de Transformados Metálicos" o "Memoria de la seguridad e industria..." entre otras importantes materias. Alguno de los más significativos puntos estudiados, iban desde la asistencia técnica, hasta los problemas urbanos, la industria de la construcción o el problema de la vivienda. El entonces ITcc, consciente de la importancia de estos viajes se implicó enormemente enviando a arquitectos de la escuela como Salustiano Albiñana, Fernando Cassinello, e ingenieros como Francisco Arredondo, Jefe de División de Materiales del ITcc, o José Ma Tobio, Doctor en Química y Jefe de Sección de Ensayos del ITcc...entre otros. Los cuales estudiaron, sobre todo, la producción de nuevos materiales, los métodos por los que se ensayaban e introducían en la práctica constructiva, las relaciones de los programas de construcción y las corrientes más modernas que imperaban en cuanto a normalización de materiales o coordinación modular además de los materiales normalizados. Para ello no solo visitaron los laboratorios especiales, en donde se analizaban las condiciones, absorción y conductividad de estos sino que también los centros de investigación de materiales americanos para observar sus valiosos métodos de ensayo e investigación (Figura 6).

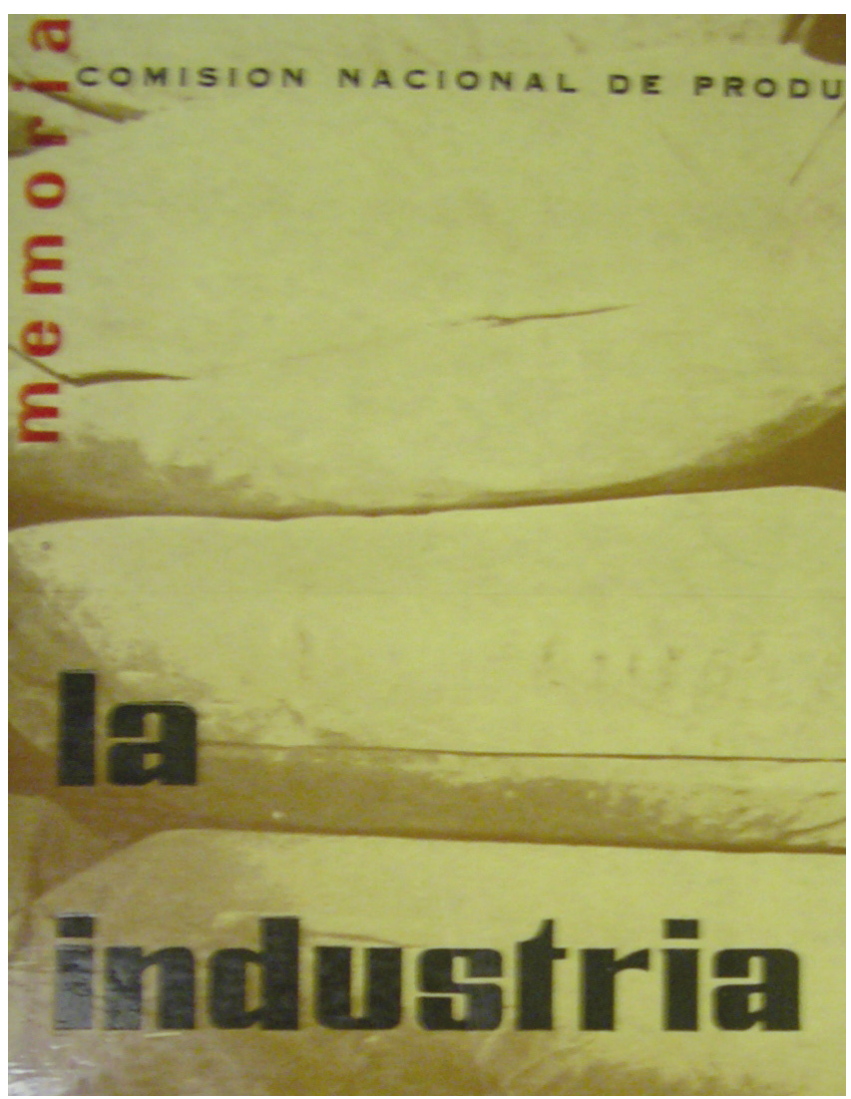

Figura 6. Memoria del viaje a EEUU. (Ministerio de Industria).

Las visitas técnicas que realizaron a instituciones como la Chicago Housing Authority, National Housing Center, International Housing Center of Washington, las Home Builders Association of Chicago, International Cooperation Administration Technical, Assistance Training Staff, American Institute of Architects, City of Los Angeles Departament of Building and Safety, New York City Housing Authority o la Associated General Constructor of American, daban idea de la trascendencia del viaje y les enseñó no solo las muchas capacidades tecnológicas de la industria americana sino cual

\footnotetext{
Otras publicaciones estadounidenses de aquellos años que se pueden encontrar en el archivo del propio Instituto de las Ciencias de la Construcción Eduardo Torroja son: American Builder, American Carpenter and Builder, American Ceramic Society Bulletin, Applied Mechanics Reviews, American Society of Mechanical Enginners, Ashrae Journal New York American Society of Heating, American Society for Testing and Materials, Ceramic Age, Concrete Products, Construction Methods and Equipment, Construction Review, Journal of Basic Engineering, Journal of Engineering Mechanics Division, Journal of Pipeline Division, Journal of the American Ceramic Society, Journal of the Construction Division, Journal of the Highway Division y World Construction.
} 
era el camino a seguir para avanzar en un anhelado progreso tecnológico ${ }^{2}$.

Mantuvieron los técnicos españoles sendas entrevistas con algunos de los arquitectos americanos más eminentes del momento: Eero Saarinen, Mies van der Rohe, Pietro Belluschi del MIT, Ludwig Hillberseimer, Louis Khan o Frank Lloyd Wright, así como las oficinas de Vincent de Ponte de I.M. Pei and Associates Architects of New York, Katz, Waisman, Blumen, Kranz, Stein y Weber de New York, Frank J. Duane, Washington Metropolitan, Ben Ronis también de Washington, Charles A. Blessing, Director City Planning Comision of Detroit, Richard J. Neutra, de Los Angeles, Rolf Skalarek de Victor Gruen and Associates, Jonh F. Carlson, William M. Shoenfeld, y Donald F. Wilcox de la importantes firma Pereira and Luckmann de Los Angeles o Andrey k. Doughty de la firma Welton Becket Associates...lo que les proporcionaría un sinfín de innovadores criterios y un cambio de comprensión en torno al conocimiento arquitectónico y constructivo (Figura 7).

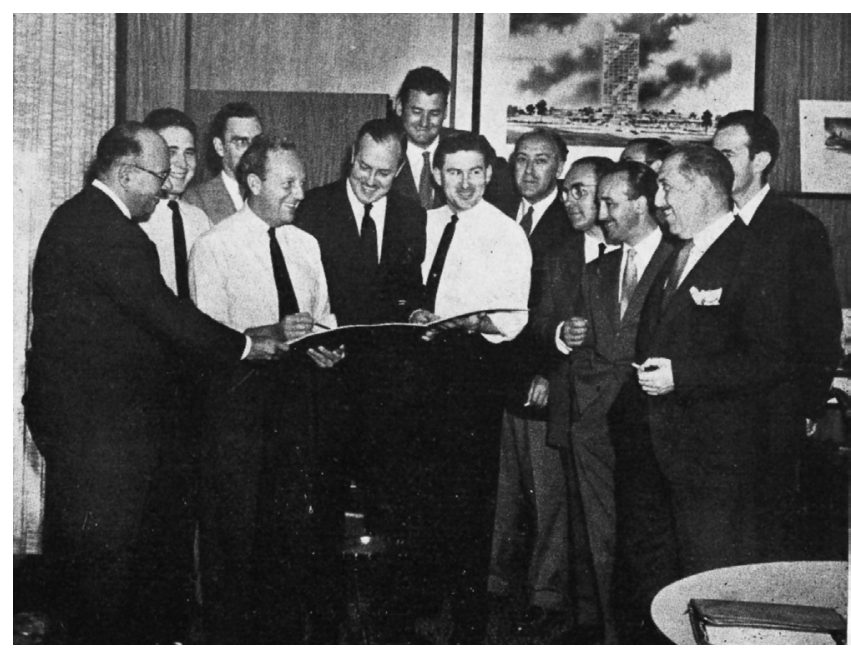

Figura 7. Arquitectos, viaje a EEUU. (Ministerio de Industria).

Los técnicos enviados por el ITcc, que tomaron parte en estos viajes a los EE.UU., se fueron familiarizando con toda una serie de conceptos como la simplicidad en la arquitectura e innovación de la ingeniería americana, adoptaron aspectos de modernidad que les vendría muy bien en su posterior labor profesional, comprendieron lo importante de aquella sistematización y normalización de todo, ya que realizaban una composición de proyectos completa, de esta forma el suministro era más óptimo en tiempo y cantidades. La trascendencia del término "efficiency" en la investigación americana lo expresaba todo (17).

Otra de las facetas más estudiada por los técnicos españoles fue la utilización de los nuevos materiales de hierro, el acero laminado, la carpintería metálica de aluminio, herrajes de mayor calidad, bloques huecos de hormigón vibrado, encofrados robustos y perfeccionados, piezas de hormigón aligerado con cámaras de aire, muros sin carga, materiales derivados del plástico, techos acústicos con fibras de vidrio, neopreno, piezas cerámicas, el vidrio doble con vacío interior, cubiertas impermeabilizadas, ascensores de gran velocidad en los rascacielos...Unidas a todo tipo de maquinarias también se valieron de los catálogos de materiales tipificados y procedimientos garantizados de sus industrias auxiliares de la construcción, que eran considerados como instrumentos de gran valía por los técnicos españoles.

Una de las ideas sostenida por estos técnicos a su vuelta fue tratar de reproducir en España lo allí estudiado, por lo que recomendaron la utilización de entre sus materiales y sistemas, aspectos tan simples como que el hormigón fuera distribuido en camiones hormigonera lo que proporcionaría una gran economía de costos y una mayor exactitud. Otros materiales a utilizar serían los ladrillos huecos pero que fueran de mayor variedad y tamaños o que se utilizaran plaquetas anti acústicas, baldosas de goma, varillas y cabillas de hierro redondo de superficie rugosa, lo que simplificaría la confección de armaduras en obras, así como las planchas de yeso prensado para construir tabiques.

Dos de los conceptos que más les sorprendió y que tratarían de implementar en sus ciudades fueron, junto a los shopping center y el entusiasmo por los rascacielos, los drive-in, que según sostuvieron algunos de los técnicos españoles que viajaron a los USA:

"The influence of the automobile in Architecture not only in parking buildings garages and so on, but also in the many "Drive in" operation such as: Drive-in Banks, drive-in theatres, drive-in restaurants, motor-hotels or motels and motor inns, drive-in deposit and many others"

Se discierne que gracias a esos viajes se produjo, -si bien las posibilidades materiales o de procedimientos eran más difíciles de cumplir al salir España de un periodo de autarquía-, más que un cambio de mentalidad, que estaba asumido, sí se profundizó en las extraordinarias consecuencias para un país que sustentaba todo su progreso industrial en los asombrosos avances científicos y tecnológicos que llegó a suscitar una altas expectativas en ciudades como Bilbao (18).

Bilbao fue la ciudad española que recibía mayores cantidades de capital americano a principios de los años 60 -más del 50\% del total-. Se suponía que aquellas elevadas inversiones traerían consigo un significativo desarrollo industrial, lo que no quiere decir que suscitase un mayor progreso científico, al hacerse tan dependientes, tal y como se corroboraría años después, de las patentes y de las tecnologías foráneas (19).

Por otra parte, aunado a estas propuestas, es preciso resaltar las consecuencias de la Fullbright Act y la implicación del ingeniero bilbaíno Leandro J. Torrontegui, ya que ambos posibilitaron que el laboratorio de la Escuela de Ingenieros de Bilbao se hiciera con valiosos materiales y equipos en desuso provenientes de algunos laboratorios de los Estados Unidos que fueron retirados, y es que dada la situación de los laboratorios bilbaínos, con un equipamiento más anticuado y

\footnotetext{
2 Otras importantes instituciones americanas con las que tomaron contacto fueron: la Office of International Labors Affaire U.S. Departament of Labor, Planning Comission of the City of New Orleans, Bureau of Reclamation U.S. Departament of the Interior, Denver Federal Center Colorado, National Association of Home Builders, National Housing Center of Washington, International Housing Service of the Housing and Home Finance Agency...
} 
escasamente dotado si los comparamos con los americanos, suscitó un inmediato progreso tecnológico. Baste recordar que la Fullbright Act, autorizaba a la Secretaria de Estado a vender material de guerra excedente y usar esos fondos en el intercambio cultural, lo que favoreció el flujo de innovadores ideas y conocimientos, suscitando una mayor investigación, desarrollo e innovación. Junto a esta propuesta llegó el Plan Marshall que suscitaría una labor, pero, en este caso, de reconstrucción material de las ciudades europeas. Plan del que también se beneficiarían las ciudades españolas (20).

Incluso los políticos y empresarios bilbaínos trataron infructuosamente durante aquellos años de hacer de Bilbao la gran metrópoli del norte, una especie de Pittsburgh, que simbolizara, a través de una serie de proyectos e ideas, lo que pretendían que fuera la capital del poder industrial y financiero de aquella España de la explosión económica. Baste recordar que una década antes en ciudades como Madrid la importancia de la influencia americana que trajeron consigo los Otamendi con su singular cultura estadounidense de ciudades como Nueva York, Chicago o Boston, y es que nos recordaba el profesor Sambricio que fue durante esos años del Desarrollismo en que la sociedad madrileña estaba deseosa de romper con su pasado y mostrar una imagen arquitectónica más moderna. Incluso cuando se refería a la influencia que hubo en las viviendas de Madrid -en varias actuaciones trataron de reproducir el estándar de vida americano- fuese una referencia obligada la arquitectura de Marcel Breuer o de Richard Neutra (Figura 8) (21) (22).

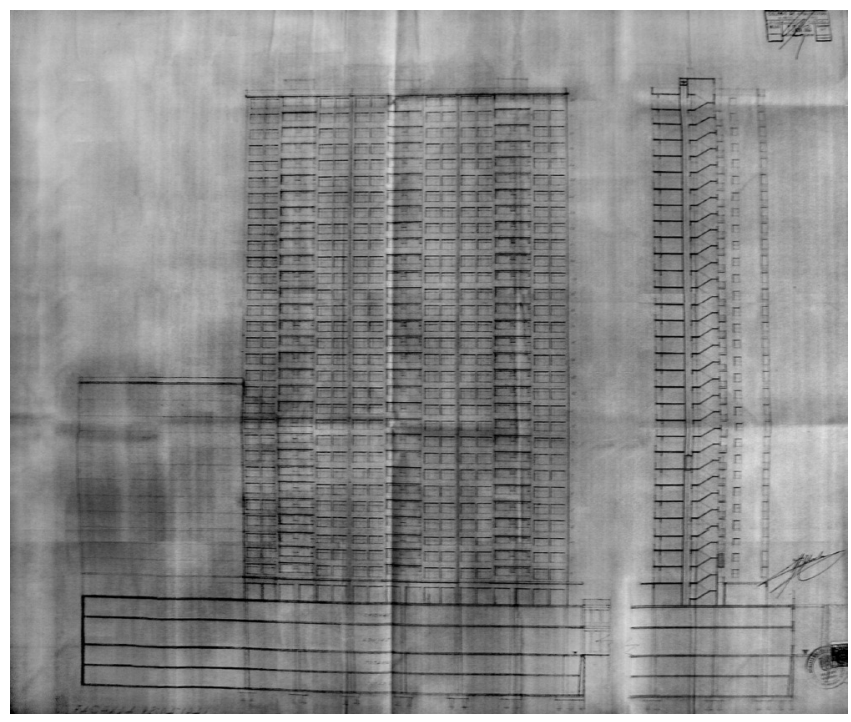

Figura 8. Proyecto rascacielos Deusto. (Archivo General de la Administración Pública de la CAE).

La influencia con aquellos viajes a los EE.UU. de la ICA y la CNPI, en los que también participaron varios arquitectos bilbaínos como Eugenio Aguinaga o Félix Iñiguez de Onzoño además de ingenieros como Antonio Zalbidegoitia, resultaron determinantes en esa pretensión de progreso tecnológico y búsqueda de modernidad. El caso de Eugenio Aguinaga fue más que significativo ya que llevó adelante dos importantes proyectos en Bilbao como consecuencia de ese viaje, como influencia de su visita a Taliesin West y tras conocer a Frank Lloyd Wright le llevó a realizar el diseño del Club de Golf de La Galea. Tras aquel viaje otra de sus propuestas americanas llegó con el proyecto de Shopping Center en las torres de
Zabalburu. Lo mismo le sucedió, tanto a José Chapa como a Enrique Casanueva y Jaime Torres, que tras hacerse con la posibilidad de diseñar el nuevo Banco de Vizcaya en Bilbao viajaron a Nueva York y a su vuelta propusieron edificar un Seagram que alzaba su fachada en bronce y cristal tratando de inmortalizar su firma o más bien un nuevo Chase Manhattan bank de Skidmore Owings y Merril, sobre el Ensanche bilbaíno. Por su parte, Javier Sada de Quinto, arquitecto del Ministerio de la Vivienda, diseñó varios rascacielos para un shopping center en Deusto además de utilizar un sistema constructivo con patente americana. A los proyectos de arquitectura se sumaban aquellos en infraestructuras como el proyecto de un puente sobre la ría del Nervión basado en el Cobo Hall de Detroit, proyecto del arquitecto José Sans Gironella, o en los túneles como el diseñado por el ingeniero Pedro Sarobe entre las dársenas de Lamiaco y Sestao basado en el Holland Tunel de Manhattan, o el proyecto de la autopista Bilbao-Behobia basado en el New York Thruway. Incluso se planteó desde la prensa escrita porque no reproducir en Bilbao el Plan Bertrand Goldberg de Chicago o por las autoridades provinciales se sustentó un gran parque de atracciones en Bilbao, en el monte Ganguren, basado en el diseño del parque de Walt Disney (Figura 9).

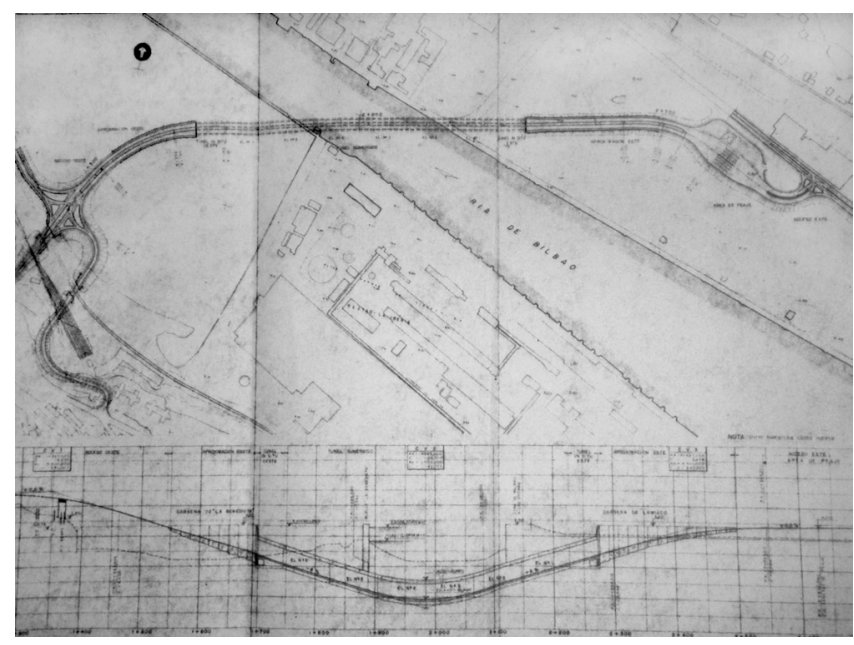

Figura 9. Proyecto túnel bajo el Nervión. (Archivo Foral de Bizkaia).

Esta estrategia tuvo, sin embargo, unas controvertidas consecuencias para la ciudad de Bilbao, dado que si bien cumplía el anhelo político empresarial de unir progreso industrial, con las directrices del IETcc de avances tecnológicos, y hacer de esta ciudad un nuevo símbolo arquitectónico y urbano, -que articularía la fisonomía de un pequeño Pittsburgh con la de Manhattan-, los efectos de aquella pretensión fueron otros bien distintos, por las numerosas críticas que suscitó en la propia sociedad bilbaína y entre los arquitectos, al sostener estos que la mera reproducción sin una base científica que la sustentara era tachada de no suponer sino una vía fracasada.

\section{A MODO DE CONCLUSIÓN}

Si la influencia que el IETcc tuvo sobre los avances que se suscitaron en el conocimiento científico y en innovaciones tecnológicas en Bilbao, durante los años del Desarrollismo, es incuestionable -al igual que en el resto de España-, porque baste recordar que, tal y como sostuvo la profesora Pepa Cassinello, con aquellos concursos, debates y congresos, que fueron respaldados por la labor científica del IETcc, estimularon 
y desarrollaron no solo el mercado industrial nacional sino que también un innovador pensamiento científico. La teoría, es evidente, que, poco o nada tuvo que ver con la pretendida praxis, porque aquellas premisas científicas sustentadas acabarían siendo incumplidas sistemáticamente -tanto por la administración pública como por la iniciativa privada- y menos aún lo serían las líneas trazadas por el IETcc, debido, -a un aspecto que era fundamental para que hubiese acaecido ese anhelado progreso-, a las exiguas inversiones económicas realizadas, que conocemos gracias a Labein o a la prensa escrita del momento (23).

A pesar de los avances suscitados, en el pensamiento científico bilbaíno, con aquellas directrices sustentadas por el IETcc, conjuntamente con las progresistas ideologías y tecnologías que llegaban de los EE.UU. y evidenciarse que sí se dieron las disposiciones necesarias para que se cumplieran dichas premisas, las consecuencias, con el transcurrir de los años, resultaron ser otras bien distintas, dando lugar a un léxico que se hizo usual: incertidumbre, incomprensión, dualidad e incluso fracaso. Una de las cuestiones que mayores discusiones suscitaba entre la propia prensa escrita bilbaína de finales de los años 70, fue, que cuando se publicaron las cifras en torno a la tecnología en España, en el año 1975, de las 10.868 patentes registradas, 9.064 lo eran de países europeos o de América del Norte, lo que evidenciaba la total dependencia tecnológica y desdecía los supuestos avances tecnológicos sostenidos por las autoridades. Constatando estos supuestos, hubo reputadas voces en el mundo de la investigación como la del profesor Salvador Pérez Arroyo quien sostuvo que no se logró reproducir una experiencia que sirviese para impulsar la tecnología española con el fin de evitar la salida de divisas por ser patentes de otros países. Incluso el también profesor Ignacio Paricio sostuvo que la importación de aquellas patentes se realizó sin criterio científico alguno.

Aquella pretensión del IETcc, desde la década de los años 60, de sustentar la necesidad de desarrollar una tecnología propia para reducir la dependencia, -a tenor de las exiguas inversiones económicas realizadas durante aquellos años-, constatas que aquellas ideas fueron en realidad denostadas e incluso relegadas, porque sucedió todo lo contrario, y es entonces cuando comprendes que la política hasta entonces desarrollada no había sino perpetuado una dependencia de la tecnología foránea. En el caso de Bilbao, el problema radicaba en que para una mayoría de sus empresarios, el tener que invertir en investigación lo consideraban un gasto infructuoso porque si no producía un beneficio inmediato no entendían la necesidad de hacerlo. Es más, la comarca del Gran Bilbao, a pesar de ser la zona más industrializada de Bizkaia, esta, al no contar con una tecnología propia, era tachada, por la misma prensa escrita y revistas locales, a finales de los años 70, de ser en realidad una zona subdesarrollada. Incluso la situación en Bilbao y en toda la provincia de Bizkaia era presentada por la propia prensa escrita local de nefasta ya que no se le dedicaba a investigación ni el o,1\% del Producto Nacional Bruto y se le criticaba, tanto a la iniciativa pública como a la iniciativa privada, de haber sostenido una línea e inversión en investigación a niveles propios de los países más subdesarrollados. La prensa los calificó, al compararlos con otros países europeos, de cifras en inversiones en investigación realmente ridículas. Es preciso recordar alguna de las premisas que aportó la OCDE que clarificaban fehacientemente aquella realidad, como que la frontera que dividía a un país desarrollado de otro que no lo era estaba constituida por el 1\% del Producto Nacional Bruto destinado a investigación. Los bajos recursos dedicados a la investigación y la no adecuación de los mismos a las necesidades de la industria vizcaína, se sumaban el envejecimiento de las estructuras productivas y, como no, sobre todo, aquella dependencia tecnológica. Baste recordar que excepto los laboratorios Torrontegui, la Universidad Pública Vasca de Lejona, el Grupo Arteche y la Asociación de Investigación de la Industria Gráfica, no existía ninguna estructura de investigación en toda la provincia de Bizkaia. Ante aquellos déficits, si aunamos la grave situación de crisis política, económica, urbana y social, de finales de los años 70, que llevó a Bilbao a lo que podemos denominar como un periodo de involución tecnológica, sirvió a un único propósito: para ocultar una singular trayectoria científica (24) (25) (26).

\section{REFERENCIAS}

(1) Neutra, R.J. (1961). Nota Necrológica sobre Eduardo Torroja. Informes de la Construcción, 14(133): $108-4$.

(2) Cassinello, F. (1961). Eduardo Torroja. Cuadernos de Arquitectura, (64), $4^{\circ}$ Trimestre.

(3) Cassinello, P. (2008, mayo). La Escuela de Eduardo Torroja: entendimiento y promoción de la innovación en la construcción arquitectónica. En AA.VV., II Jornadas de Investigación en Construcción. Instituto de las Ciencias de la Construcción Eduardo Torroja/CSIC, Madrid.

(4) Esteban-Maluenda, A. (2008). Informes de la Construcción: más que una revista técnica. Informes de la Construcción, 6o(510): 87-102, doi: http://dx.doi.org/10.3989/ic.2008.v6o.i510.

(5) Nadal, J. (1964). La evolución de la industria de la construcción. Informes de la Construcción, 17(161): 3-12.

(6) Nadal, J. (1964). Comentarios a propósito de la segunda conferencia de parlamentarios y científicos conjuntamente con el consejo de Europa y la OCDE. Informes de la Construcción, 17(162): 3-6.

(7) Pastor, J. (1964). La dinámica de los procesos industriales. Patronato de los Laboratorios de Ensayos e Investigación Industrial Leandro Torrontegui.

(8) Oroviogoicoechea, A. (1961). El departamento de Construcción de los Laboratorios de Ensayos e Investigación Industrial "Leandro José Torrontegui". Dyna, Ingeniería e Industria, 36(7): 476-487.

(9) AA.VV. (1966). Ensayos e Investigación, 1(1). Bilbao.

(10) Gertrudis, P. (1966, 13 de agosto). Arquitectura una ciencia al servicio del hombre. Hierro.

(11) Del Busto, J.A. (1966, 10 de septiembre). Viviendas modestas necesidad urgente. Hierro.

(12) Maria, M. (1970, 9 de noviembre). La vivienda en el mundo. Hierro.

(13) Barrena, C. (1959, 15 de agosto). Más de 75.00o personas han visitado ya los pabellones del certamen. Diario Oficial de la Feria de Muestras de Bilbao. 
(14) Ayuntamiento de Bilbao. (1970). Feria de Muestras de Bilbao. Boletín de Estadística de la Villa de Bilbao, $\mathrm{n}^{\circ} 659$.

(15) Fauquie, F., Bellosillo, F.J. (1979). Roma Interrotta, incontro internazionale d' arte. Comun, $\mathrm{n}^{0} 1$.

(16) López-Otero, M. (1961). Eduardo Torroja y los arquitectos. Arquitectura, $\mathrm{n}^{\mathrm{O}} 31$.

(17) Bilbao, L. (2006). El debate en torno a la influencia de la arquitectura estadounidense en España: Los arquitectos Luis Vázquez de Castro, Valentín Picatoste y las memorias de los técnicos españoles en los EE.UU. En La arquitectura norteamericana, motor y espejo de la arquitectura española en el arranque de la modernidad (1940-1965), (pp. 81-86). Pamplona: ETSAN, T6 Ediciones.

(18) F.B. (1957, 25 de abril). Arquitectos españoles en los Estados Unidos. Los viajes de estudio en el plan americano de ayuda exterior. El Correo Español/El Pueblo Vasco.

(19) Areilza, J.M. (1959). Presente y futuro de las relaciones económicas entre España y los EE.UU. Información, $\mathrm{n}^{\mathrm{0}} 1042$.

(20) Labein (2005). Labein la fuerza de una idea, 50 años de progreso. Bilbao: Labein-Tecnalia.

(21) Sambricio, C. (2009). CUN, CIM y VACESA, 1939-1955. En La construcción de la ciudad liberal. Madrid: Ricardo S. Lampreave.

(22) Sambricio, C. (2004). La vivienda en Madrid en la década de los cincuenta. En Madrid, vivienda y urbanismo 19oo1960, Madrid: Ediciones Akal.

(23) Cassinello, P. (2000). Razón científica de la modernidad española en la década de los 50. En Los años 50, la arquitectura española y su compromiso con la historia, (pp. 21-38).Congreso Internacional ETSAN, Pamplona: T6 Ediciones.

(24) Pérez-Arroyo, S. (1976). Artesanía e industrialización del alojamiento de masas. Arquitectura, $\mathrm{n}^{\circ} 199$.

(25) Paricio, I. (1980). La crisis de la prefabricación. Cuadernos de Arquitectura y Urbanismo, $\mathrm{n}^{\circ} 48$.

(26) Heros, A. (1978, 23 de octubre). La investigación en Vizcaya a niveles africanos. Hierro. 